\title{
Combined microbiological test to assess changes in an organic matrix used to avoid agricultural soil contamination, exposed to an insecticide
}

\author{
G.R.Tortella, 1,3*, E. Salgado ${ }^{3}$, S.A. Cuozzo ${ }^{2}$, R.A. Mella-Herrera ${ }^{3}$, L. Parra ${ }^{3}$, M.C. \\ Diez, ${ }^{1.3}$.and O. Rubilar ${ }^{1,3}$
}

${ }^{1}$ Facultad de Ingeniería Ciencias y Administración, Departamento de Ingeniería Química, Universidad de La Frontera, Temuco, Chile.

*Corresponding author: gonzalo.tortella@ufrontera.cl

${ }^{2}$ Planta Piloto de Procesos Industriales Microbiológicos (PROIMI-CONICET), , T40001MVB, Tucumán, Argentina. Universidad Nacional de Tucumán.

${ }^{3}$ Scientific and Technological Bioresource Nucleus, Universidad de La Frontera, Temuco, Chile.

\begin{abstract}
Combined microbiological and molecular test (BiologEcoplate ${ }^{\mathrm{TM}}$, denaturing gradient gel electrophoresis (DGGE) and Real Time PCR (qPCR)) were carried out to evaluate the impact of repeated diazinon (DZN) applications at high concentration $\left(40 \mathrm{mg} \mathrm{kg}^{-1}\right)$ on microbial communities in a microcosm simulating the organic matrix (straw $(50 \%)$ : peat $(25 \%)$ : soil $(25 \%) \mathrm{vv}^{-1}$ ) of a pesticide biopurification system (PBS). Pesticide dissipation was also evaluated. After three successive exposures with DZN the dissipation efficiency was high; DZN dissipation clearly accelerated in the organic matrix, achieving $87 \%, 93 \%$ and $96 \%$ respectively after the three applications. The results obtained with BiologEcoplate ${ }^{\mathrm{TM}}$ showed that the physiological profiles of the community were not affected by the addition of DZN. On the other hand, molecular assays (DGGE and qPCR) demonstrated that the microbial structure (bacteria and fungi) remained relatively stable over time with high DZN doses compared to control. The results of the present study clearly demonstrate the high dissipation capacity of this biomixture and highlight the microbiological robustness of this biological system.
\end{abstract}

Keywords: CLFP, biopurification system, microbial communities, diazinon. 


\section{Introduction}

Pesticide biopurification systems (PBS), also known as biobeds, are a biotechnological tool applied in on-farm bioprophylaxis systems to treat pesticide residues produced by point source contamination due to inadequate pesticide handling in agricultural activities. PBS are widely used in European and South American countries (Castillo et al., 2008). They consist of several components including an organic matrix, typically composed of straw, peat and soil (2:1:1 by volume), in which pesticides are degraded (Torstensson and Castillo, 1997; Castillo et al., 2008). In some countries, such as Italy, Greece and France, this organic matrix has been adapted to local conditions, varying the types and proportions of lignocellulosic substrates (Coppola et al., 2007; Castillo et al., 2008; Karanasios et al., 2010). Efficient degradation of both single pesticides and mixtures have been reported using traditional and modified organic matrices (Fogg et al., 2003; Coppola et al., 2007; Vischetti et al., 2008; Karanasios et al., 2010; Tortella et al., 2010; Fernandez- Alberti et al., 2012; Tortella et al., 2012), including fullscale model biobeds (Spliid et al., 2006; Omirou et al., 2012).

From a microbiological point of view, studies have been performed to evaluate the effect of pesticides on microbial communities and biological activities in the organic matrix. However, most of these studies were carried out using a modification of the typical composition with straw, peat and soil (Vischetti et al. 2007; Vischetti et al., 2008; Coppola et al., 2011). Although PBS with the typical organic matrix are widely distributed (Castillo et al., 2008), less information is available for understanding the interactions and the effects of pesticides in this organic matrix from the microbiological point of view. This is important for ensuring the long term sustainability of this biopurification system. Various studies have been done on the traditional biomixture composed of straw, peat and soil. Sniegowski et al. (2011) reported that no changes were observed in microbial communities in the biomixture when exposed to linuron. However, some variations were detected in microbial communities due to environmental changes such as cold or a period of drought. More recently Tortella et al. (2013 $\mathrm{a}, \mathrm{b})$ reported that the fungicide carbendazim and the herbicide atrazine applied repeatedly in a traditional biomixture (straw:peat:soil) caused inhibitory effects on microbial activities as revealed by several enzymatic activities and BiologEcoplate ${ }^{\mathrm{TM}}$. However, these inhibitory effects were transitory and no negative effects were observed in pesticide degradation. Moreover, these authors reported that no alterations in microbial diversity were observed using DGGE. As mentioned above, the effects of several pesticides on microbial communities in the organic matrix (typical or modified) have been evaluated. However, these investigations have been restricted to studies with herbicides and fungicides. The present work investigates the effects of insecticides, which are widely used in agricultural activities but have not been extensively studied.

Diazinon (O,O-diethyl O-(2-isopropyl-6methylpyrimidin-4-yl) phosphorothioate) is a nonsystemic organophosphate insecticide formerly 
used to control a wide range of insects. Diazinon has been widely used for indoor and agricultural pest control in concentrations between 2 and 10 $\mathrm{mg}$ of active ingredient per kilogram (mg a.i. $\mathrm{kg}^{-}$ 1) to control a number of insects which affect food production (Cycón et al. 2010). Residential uses of diazinon were outlawed in the U.S. in 2004 but it is still approved for agricultural uses. Diazinon was therefore used as an insecticide model in this study. In the present work we combined molecular techniques (DGGE and qPCR) with physiological profiles using BiologEcoplate ${ }^{\mathrm{TM}}$ to evaluate the impact of diazinon, applied repeatedly at high concentrations, on microbial functional diversity and microbial communities in a microcosm simulating the traditional organic biomixture in biobed systems.

\section{Materials and Methods}

\subsection{Chemicals}

Analytical standard diazinon (DZN) (99\% purity) was purchased from Sigma Aldrich, Chile. Commercial formulation DZN (diazinon 40 WP) was supplied by Anasac Chile S.A. MBTH (3-methyl-2-benzothiazolinone hydrazone) and DMAB (3-(dimethylamino) benzoic acid) were purchased from Sigma Aldrich, Chile. All other chemicals and solvents were of analytical reagent grade and were purchased from Equilab Ltda and Merck S.A (Chile).

\subsection{Organic matrix preparation}

The organic matrix was prepared by mixing soil, commercial peat (36.6\% organic carbon) and winter wheat straw (34.0\% organic carbon) in the volumetric proportion of $25: 25: 50 \mathrm{vv}^{-}$ 1. Soil was collected from the Maquehue experimental station (Andisol Freire series; $38^{\circ} 50^{\prime}$ S, $72^{\circ} 41^{\prime}$ W) of La Frontera University; this site had no history of DZN use and the principal soil characteristics are shown in Table 1. The constituents were processed and mixed as previously reported in Tortella et al. (2013a)

Table 1. Characteristics of soil used in the biomixture.

$\begin{array}{lr}\text { Parameters } & \text { Value } \\ & \\ \text { Sample depth (cm) } & 0-20 \\ \mathrm{~N}\left(\mathrm{mg} \mathrm{kg}^{-1}\right) & 36 \\ \mathrm{P}\left(\mathrm{mg} \mathrm{kg}^{-1}\right) & 12 \\ \mathrm{~K}\left(\mathrm{mg} \mathrm{kg}^{-1}\right) & 751 \\ \mathrm{pH}(\text { water }) & 6.1 \\ \text { Organic matter (\%) } & 18 \\ \text { Sand }(\%) & 30.8 \\ \text { Silt }(\%) & 41.8 \\ \text { Clay (\%) } & 27.4\end{array}$

\subsection{Experimental design and diazinon treatments}

Bulk samples $(2.0 \mathrm{~kg})$ of organic matrix were placed in glass containers ( $40 \times 20 \times 10 \mathrm{~cm}$ deep) and the contaminant (40 $\left.\mathrm{mg} \mathrm{kg}^{-1}\right)$ was applied following the protocol previously described in Tortella et al. (2013a). DZN was applied successively three times (days 0, 30 and 60) and diluted with sterilized distilled water in a predetermined volume to obtain biomixture moisture of approximately $40 \%$. Organic matrix without DZN was established as control and received the same volume of sterile distilled 
water as the treatment with DZN. All the glass containers were covered with perforated plastic film to avoid excessive evaporation and incubated in the dark at $25 \pm 2{ }^{\circ} \mathrm{C}$ for a period of 90 days. The organic matrix moisture was kept stable by regularly adding sterile distilled water. Samples were collected at fixed intervals to determine residual DZN and microbiological parameters. Residual DZN was extracted from $10 \mathrm{~g}$ of the biomixture with $30 \mathrm{~mL}$ of acetonitrile of HPLC grade ( $2 \mathrm{~h}$ shaking at $350 \mathrm{rpm}$ and $30 \mathrm{~min}$ of ultrasonication). The samples were then centrifuged $(13,500 \times \mathrm{g})$, and the resulting supernatant was filtered using a PTFE membrane (0.2 $\mu \mathrm{m}$ pore size; Millipore) and analysed by high performance liquid chromatography (HPLC). The extraction technique was validated by contamination of the biomixture samples with DZN at dry weights of 1,10 and $40 \mathrm{mg}$ $\mathrm{kg}^{-1}$. The average recoveries (\%) after DZN addition were $90 \pm 1.2 \%, 94.7 \pm 1.9 \%$ and $94.8 \pm$ $1.2 \%$, respectively. The DZN degradation in the biomixture followed first-order kinetics, and the DZN concentration at a given post-application time $(\mathrm{t})$ could be described by the equation $\mathrm{C}$ $=\mathrm{C}_{0} \mathrm{e}^{-\mathrm{kt}}$. The half-life of DZN was determined using the equation $t_{1 / 2}=\operatorname{Ln}(2) / k$.

\subsection{Microbiological analysis}

\subsubsection{Community level physiological profiles}

Community level physiological profiles (CLPPs) were assessed by the BiologEcoplate ${ }^{\mathrm{TM}}$ system (Biolog Inc., CA, USA). Microplates containing 96 wells with 31 sole carbon sources and a water blank, all in triplicate, were inoculated according to the methodology proposed by Fang et al. (2009) with some modifications as previously described in Tortella et al. (2013b).

The microbial activity of each microplate was expressed as the average well-colour development (AWCD) and was determined as previously described by Garland and Mills (1991) as follows: $\mathrm{AWCD}=\sum \mathrm{OD}_{i} / 31$, where $\mathrm{OD}_{i}$ indicates the optical density of substrate $i$ divided by the lowest optical density, measured in the water blank well at $590 \mathrm{~nm}$. Furthermore, the biomixture diversity index was calculated as described previously by Fang et al. (2009), as follows:

$\begin{array}{lll}\text { a) Shannon-Weaver index } & \mathrm{H}=\sum p_{i}(\ln \\ \left.p_{i}\right) & \\ \text { b) Shannon Evenness index } & \mathrm{E}=\mathrm{H} / \ln \mathrm{S}\end{array}$

Here, $p_{i}$ is the proportional colour development of the $i^{\text {th }}$ well over the total colour development of all the wells. $\mathrm{S}$ is the number of wells with colour development (substrate utilisation richness).

\subsubsection{Bacterial and fungal communities analyzed by PCR-DGGE}

The total DNA from the organic matrix $(0.4 \mathrm{~g})$ was extracted from each glass container using the NucleoSpin Soil DNA Isolation Kit (MachereyNagel. GmbH \& Co., Germany) according to manufacturer's instructions at days $0,10,30,40$, 60,70 and 90 .

The bacterial DNA was amplified with primers F341 (5' - CCTACGGGAGGCAGCAG) with a GC clamp(5'-CGCCCGGGGCGCGCCCCGGG CGGGGCGGGGGCACGGGGGG) attached to 
its 5'end and R534 (ATTACCGCGGCTGCTGG) (Muyzer et al., 1993), which target the 16S rRNA gene. Positive (Pseudomonas spp.) and negative controls were run for every set of PCRs. ITS regions of the fungal rRNA gene were amplified using the primers ITS3 (5'-ATCGATGAAGAACGCAGC) with a GC clamp(5' -CGCCCGGGGCGCGCCCCGGGC GGGGCGGGGGCACGGGGGG) and ITS4 (5'-TCCTCCGCTTATTGATATGC) (White et al., 1990). Positive (Anthracophyllum discolor) and negative controls were run for every set of PCRs. All PCR conditions are described in previous work (Tortella et al., 2013b). The cluster analysis of DGGE banding profiles was carried out using the Phoretix 1D analysis software (Nonlinear Dynamics, Durham, USA). The similarity in the profiles of the bands was calculated on the basis of the Pearson correlation coefficient with the UPGMA (Unweighted Pair Group Method with Arithmetic Mean) clustering algorithm.

\subsubsection{Quantitative PCR analysis}

The reactions used to determine the relative abundance of fungal and bacterial ribosomal gene copy numbers were performed with a StepOnePlus Real-Time PCRT System (Applied Biosystems) and HOT FIRE Pol ${ }^{\circledR}$ EvaGreen ${ }^{\circledR}$ qPCR Mix Plus. Bacterial 16S rRNA copy numbers were estimated using set Eub338 (5'ACTCCTACGGGAGGCAGCAG) and Eub518 (5' ATTACCGCGGCTGCTGG) (Muyzer et al., 1993). Fungal 16S rRNA copy numbers were estimated using set FR1 (5' AICCATTCATCGGTAIT) and FF390 (5' CGATAACGAACGAGACCT) (Vainio and Hantula, 1999). PCR amplifications used the following program: $95{ }^{\circ} \mathrm{C}$ for $2 \mathrm{~min} ; 40$ denaturation cycles at $95{ }^{\circ} \mathrm{C}$ for $15 \mathrm{~s}$, annealing at $63{ }^{\circ} \mathrm{C}$ (bacteria) and $58{ }^{\circ} \mathrm{C}$ (fungi) for $20 \mathrm{~s}$, and extension at $72{ }^{\circ} \mathrm{C}$ for $20 \mathrm{~s}$. Each $20 \mu \mathrm{L}$ reaction contained $4 \mu \mathrm{L}$ of EvaGreen ${ }^{\circledR}, 0.6$ $\mu \mathrm{L}$ of each primer $(10 \mu \mathrm{M}), 9.8 \mu \mathrm{L}$ sterilized milli-Q water, and $2 \mu \mathrm{L}$ template DNA (0.5 ng $\left.\mu \mathrm{L}^{-1}\right)$. Standard curves $\left(r^{2}>0.995\right)$ were generated using triplicate 10 -fold serial dilution of a plasmid containing a full-length copy of either the Escherichia coli 16S rRNA gene or the Anthracophyllum discolor 16S rRNA gene.

\subsection{Chemical analysis}

The DZN levels in the biomixture were measured by HPLC using a Merck Hitachi L-2130 pump, a Rheodyne 7725 injector with a $20 \mu \mathrm{L}$ loop and a Merck Hitachi L-2455 diode array detector. Separation was achieved with a C18 column (Chromolit RP-18e, 5 m 4.6 x 100 mm). Eluent A was $1 \mathrm{mM}$ ammonium acetate, and eluent $\mathrm{B}$ was acetonitrile. The flow rate was set at $1.0 \mathrm{~mL}$ $\mathrm{min}^{-1}$, which is $0-10 \mathrm{~min}$ in isocratic mode. The column temperature was maintained at $30^{\circ} \mathrm{C}$. The detector was set at $246 \mathrm{~nm}$ for data acquisition. Detection time for DZN was $3 \mathrm{~min}$. Instrument calibrations and experimental quantifications were performed against pure reference standards (0.1-10 $\left.\mathrm{mg} \mathrm{L}^{-1}\right)$ of DZN.

\subsection{Statistical analysis}

Experiments were conducted using completely randomized block design with three independent replicates. Data were subjected to a one-way analysis of variance (ANOVA) and the averages were compared by Duncan's multiple range tests with a 0.95 confidence level. 


\section{Results and Discussion}

\subsection{Diazinon dissipation in the biomixture}

After repeated DZN applications to the organic matrix, rapid dissipation of the insecticide is observed as shown in Figure 1. Residual DZN levels of around 13\%, $7 \%$ and $4 \%$ were found 30 days after the first, second and third applications respectively. Interestingly, DZN dissipation in the organic matrix accelerated, as shown in Figure 1. The kinetic data derived from the first-order function (Figure 1) also showed that DZN dissipation increased remarkably, with a dissipation rate constant that changed from 0.06 to $0.14 \mathrm{~d}^{-1}$ and a $\mathrm{t}_{1 / 2}$ that ranged from 10.8 to 4.9 days after the first and third applications respectively. This is a normal process that has been observed previously in soil contaminated with pesticides after repeated applications (Reed et al., 1987; Yu et al., 2009; TrikyDotan et al., 2010), suggesting that the microorganisms present in the organic matrix adapt to the pesticide and metabolise it at an increasing rate. However, this phenomenon is not restricted to soil; accelerated pesticide dissipation has been also observed in biobed systems. Vischetti et al. (2008) reported that metalaxyl (fungicide) was rapidly degraded in an organic matrix composed of vine/branches and urban waste/garden compost. They found that with three successive applications the half-life $\left(t_{1 / 2}\right)$ was reduced from 37 days after the first application to 4 days after the third. Recently, Tortella et al. (2013b) reported that accelerated degradation of carbendazim (fungicide) was observed in a traditional biomixture after 3 repeated applications of the pesticide at $40 \mathrm{mg} \mathrm{kg}^{-1}$. These authors reported that the half-life $\left(t_{1 / 2}\right)$ was reduced from 10 days to 4 after three successive applications of the fungicide This finding is important for the biobed system, because rapid pesticide degradation in the organic matrix is desired in this biological system.

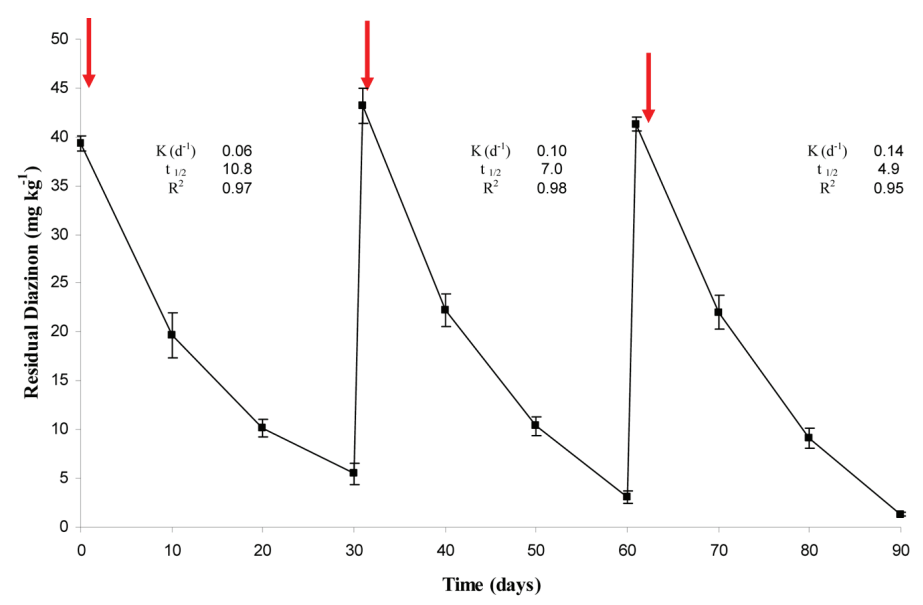

Figure 1. Residual diazinon in the biomixture after repeated applications at $40 \mathrm{mg} \mathrm{kg}^{-1}$, incubated at $25 \pm 2{ }^{\circ} \mathrm{C}$ during 90 days. Arrows indicate days when diazinon was applied. 


\subsection{Community level physiological profiles}

As shown in Figure 2, no significant $(p<0.05)$ inhibitory effect on microbial functional diversity was found due to the presence of DZN in the organic matrix. The same increase in the average well colour development (AWCD) was detected for DZN treated samples and the control during the 90 days of incubation. Changes in AWCD can indicate that the microbial population in the biomixture has changed over time. However, the results reported here demonstrate that high doses of DZN $\left(\leq 40 \mathrm{mg} \mathrm{kg}^{-1}\right)$ could be added to the organic matrix without causing important changes either in its physiological profile or in pesticide dissipation, which suffered no negative effects as demonstrated in Figure 1 above. In a contrasting result, Tortella et al., (2013 a, b) reported that the application of atrazine and carbendazim to an organic matrix composed of straw, peat and soil caused significant inhibitory effect on microbial functional diversity. However, the results demonstrated that the effects of these pesticides were not irreversible, and initial AWCD values were recovered after 90 days of incubation and three successive applications of the two pesticides.

On the other hand, the results presented in Figure 3 clearly demonstrate that the richness and homogeneity of the microorganisms in DZN-treated samples, assessed by Shannon's diversity index $(H)$ and Shannon's evenness index (E) respectively, were not significantly altered by repeated applications of DZN. The values for $\mathrm{E}$ (ranging from 0.94 to 0.97 ) indicate that the microbial communities in the DZNtreated organic matrix were stable and that the balance between the different microorganisms was not altered. These results disagree with the findings of Tortella et al. (2013a), that successive applications of atrazine in high dosage caused changes in microbial diversity indices in the biomixture of biobed systems. From this it may be deduced that the chemical nature of the pesticides could cause different responses in microbial communities in the biomixture.
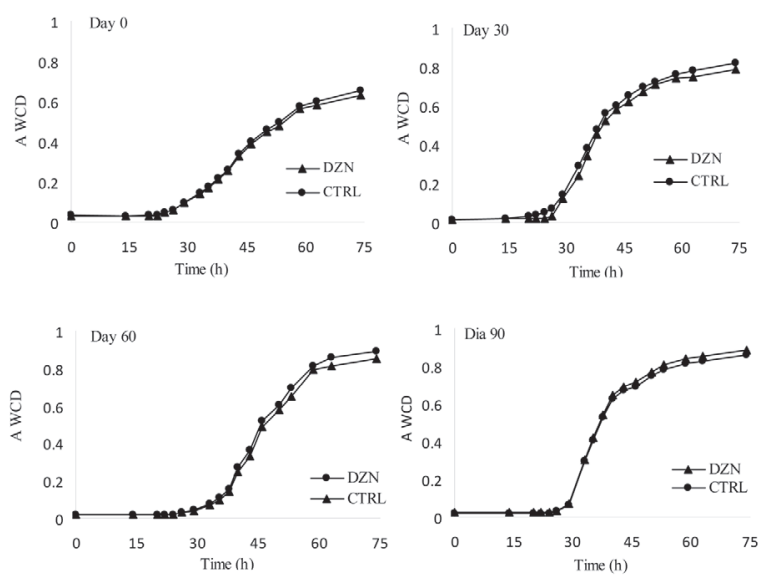

Figure 2. Average well color development (AWCD) of biomixture samples at different sampling times after repeated applications of diazinon $\left(40 \mathrm{mg} \mathrm{kg}^{-1}\right)$. (Controls (CRTL) and diazinon treatments $(\mathrm{DZN}))$. 

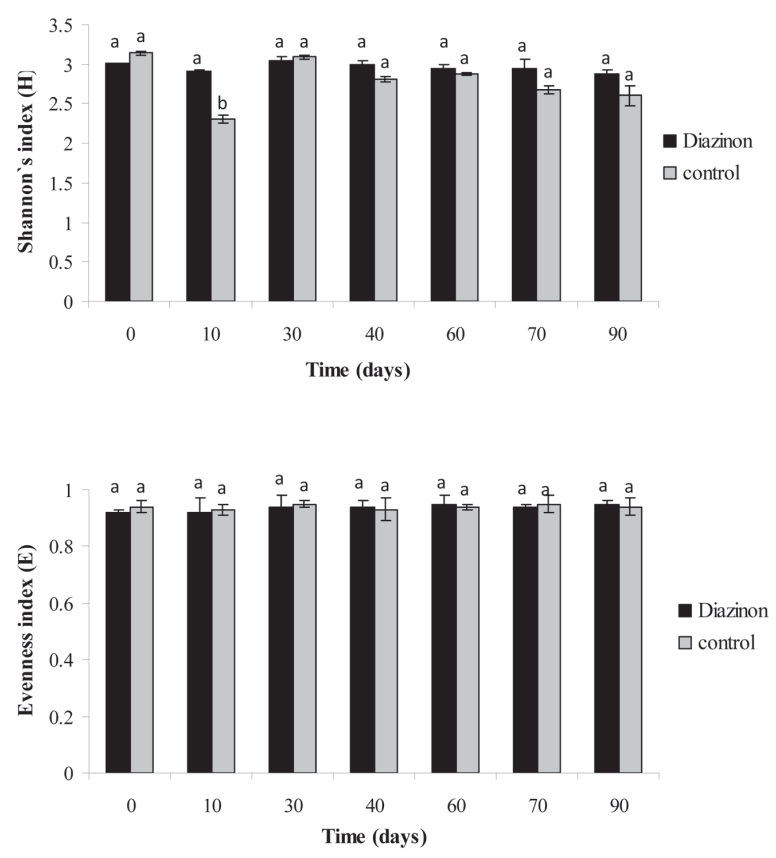

Figure 3. Diversity indices of biomixture microbial communities after repeated applications of diazinon (40 $\left.\mathrm{mg} \mathrm{kg}^{-1}\right)$. Different letters show significant differences between diazinon and control at the same sampling point. Mean values $(n=3)$ with Duncan Test $(p \leq 0.05)$.

\subsection{DGGE profiles}

After three successive applications of DZN $\left(40 \mathrm{mg} \mathrm{kg}^{-1}\right)$, the structures of the bacterial and fungal communities were assessed by denaturing gradient gel electrophoresis (DGGE). As shown in Figure 4, DGGE lanes for DZN-treated samples and controls were identified as Dzn 0 to Dzn 90 and Ctrl 0 to Ctrl 90 respectively, where the number indicates the sampling day.

The results found in the DGGE profiles for the microbial communities showed equally intense sets of DGGE bands for fungi and bacteria (Figure 4). The presence of a large number of equally abundant ribotypes for bacteria
(Figure 4a) and fungi (Figure 4c) can be clearly observed for both DZN-treated samples and control. On the other hand, UPGMA clustering revealed the existence of several groups in each type of microorganism. Nevertheless, a high similarity ( $>80 \%$ ) was found between these groups, indicating that the insecticide DZN could produce minimal changes in the bacterial and fungal structures in the organic matrix. Sniegowski et al. (2011) reported that no changes were observed in microbial communities in the organic biomixture of a biobed system exposed to linuron. However, some variations were detected in microbial communities due to environmental changes such as cold or a period of drought. Moreover, the changes observed 
were attributed to variations in specific linurondegrading microorganisms.

The information provided by the DGGE patterns obtained in this study demonstrates that microbial communities in the biomixture treated with successive doses of DZN at $40 \mathrm{mg}$ $\mathrm{kg}^{-1}$ remained relatively unchanged over time. The results reported in this study are consistent with the results reported by Tortella et al. (2013 b, c) where the microbial structures of bacteria, fungi and actinobacteria accessed by DGGE after repeated applications of atrazine or carbendazim at $40 \mathrm{mg} \mathrm{kg}^{-1}$ were unchanged after 90 days of incubation. Similar results have been reported also in studies carried out with compost-based biomixtures. Results reported by Coppola et al. (2011) showed that the successive application of different vineyard fungicides caused changes in microbial DGGE profiles. However, these changes were only temporary. In another study, Marinozzi et al. (2012) reported that repeated applications of the fungicides azoxystrobin, penconazole and fludioxonil caused only a transitory effect on microbial parameters evaluated, with full recovery 60 days after pesticide application. According to the results reported here and those obtained in previous assays with traditional biomixture (Tortella et al. 2013b, c), it may be said that this biomixture showed great robustness from a microbiological viewpoint, and that only minimal changes occur after repeated pesticide applications. However, it is important to note that changes in overall microbial communities in the biomixtures of biobed systems assessed by DGGE have been attributed to other factors than pesticide application (Sniegowski et al., 2011).

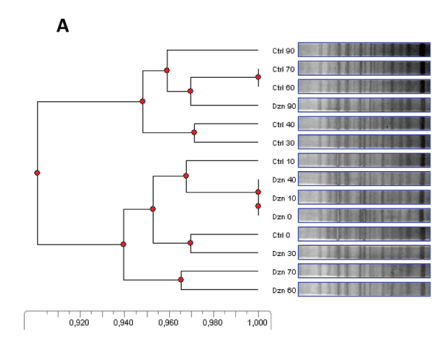

C

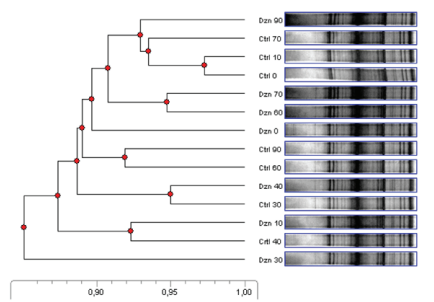

B

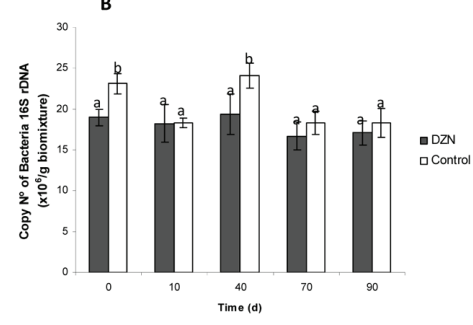

D

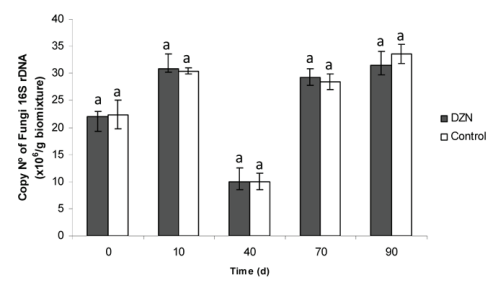

Figure 4. Cluster analysis of (A) bacterial and (B) fungal amplicons from PCR-DGGE profiles and 16S rDNA copy numbers for bacteria (B) and Fungi (D) from qPCR analysis after repeated applications of diazinon (DZN) at $40 \mathrm{mg} \mathrm{kg}^{-1}$. DNA was isolated at $0,10,30,40,60,70$, and 90 days (Controls (Crtl) and diazinon treatments (Dzn)). 


\subsection{Quantitative PCR analysis}

In general, no changes in the size of the bacterial and fungal populations of the organic matrix expressed as 16S rDNA gene, determined by qPCR assays, can be observed between DZNtreated biomixture and the control (Figure 4). A decrease in the size of bacterial populations can be observed at 0 and 40 days (Figure 4a). However, this decrease cannot be attributed to the presence of DZN, because a clear reduction in control samples in general was observed for all incubation periods. Similar results were found in fungal communities (Figure 4d), where no significant differences were found $(p<0.05)$ between DZN-treated samples and the control after three successive applications of pesticide. On the other hand, an important decrease in the 16S rDNA copy number for fungi was observed in both the control and the treated samples. This could be explained by a reduction in easily bioavailable carbon in the biomixture as reported by Tortella et al $(2013 \mathrm{a}, \mathrm{b})$. Is important to note that the reductions in the bacterial and fungal populations were not expressed as reductions in diversity, as was confirmed by the Shannon Diversity and Evenness indices obtained in this work, and the high similarity between the samples obtained by DGGE assays.

\section{Conclusion}

The organic matrix presented a high capacity for DZN dissipation, and was demonstrated that the half-life of this compound was reduced after each application, indicating a possible adaptation of microbial communities to the presence of pesticide. Physiological profiles using BiologEcoplate and molecular analysis by
DGGE and qPCR demonstrated that microbial communities in the organic matrix were stable after each application of DZN, supporting the idea that the biomixture evaluated here has a high capacity for dissipation, but also demonstrating the great robustness of this bioprophylaxis system from a microbiological viewpoint. Both this study and previous works demonstrate great microbiological robustness and capacity for pesticide dissipation. However, further investigation is necessary to evaluate the influence of high doses of herbicides, insecticides and fungicides in combination on the microbiological parameters, which could affect the efficiency of this biopurification system.

\section{Acknowledgements}

This work was supported by DIUFRO project DI14-1003, FONDECYT project No 11100236 and CRHIAM/CONICYT/FONDAP15130015

\section{References}

Castillo, M.d.P., Stenstrom, J., Ander, P. 1994. Determination of manganese peroxidase activity with 3-methyl-2-benzothiazolinone hydrazone and 3-(dimethylamino)benzoic acid. Anal of Biochemistry. 218, 399-404.

Castillo, M.d.P., Torstensson, L. 2007. Effect of biobed composition, moisture, and temperature on the degradation of pesticides. Journal of Agricultural and Food Chemistry. 55, 5725-5733.

Castillo, M.d.P., Torstensson, L., Stenstrom, J. 2008. Biobeds for environmental protection from pesticide use--a review. Journal of Agricultural and Food Chemistry. 56, 62066219. 
Coppola, L., Castillo, M.D., Monaci, E., Vischetti, C. 2007. Adaptation of the biobed composition for chlorpyrifos degradation to southern Europe conditions. Journal of Agricultural and Food Chemistry. 55, 396401.

Coppola, L., Comitini, F., Casucci, C., Milanovic, V., Monaci, E., Marinozzi, M., Taccari, M., Ciani, M., Vischetti, C. 2011. Fungicides degradation in an organic biomixture: impact on microbial diversity. New Biotechnology. 29, 99-106.

Cycoń, M., Piotrowska-Seget, Z., Kozdrój, J. 2010. Microbial characteristics of sandy soils exposed to diazinon under laboratory conditions. World Journal Microbiology and Biotechnology. 26, 409-418.

Fang, H., Yu, Y.L., Chu, X.Q., Wang, X.G., Yang, X.E., Yu, J.Q. 2009. Degradation of chlorpyrifos in laboratory soil and its impact on soil microbial functional diversity. Journal of Environmental Science-China. 21, 380-386.

Fernández-Alberti, S, Rubilar, O, Tortella, G.R, Diez, M.C. 2012. Chlorpyrifos degradation in a Biomix: Effect of pre-incubation and water holding capacity. Journal of Soil Science and Plant Nutrition, 12(4), 785-799.

Fogg, P., Boxall, A.B.A., Walker, A., Jukes, A.A. 2003. Pesticide degradation in a 'biobed' composting substrate. Pesticide Management Science. 59, 527-537.

Karanasios, E., Tsiropoulos, N.G., Karpouzas, D.G., Menkissoglu-Spiroudi, U. 2010. Novel biomixtures based on local Mediterranean lignocellulosic materials: evaluation for use in biobed systems. Chemosphere. 80, 914921.
Karanasios, E., Tsiropoulos, N.G., Karpouzas, D.G. 2012. On-farm biopurification systems for the depuration of pesticide wastewaters: recent biotechnological advances and future perspectives. Biodegradation. 23, 787-802.

Marinozzi, M., Coppola, L., Monaci, E., Karpouzas, D.G., Papadopoulou, E., Menkissoglu-Spiroudi, U., Vischetti, C. 2012. The dissipation of three fungicides in a biobed organic substrate and their impact on the structure and activity of the microbial community. Environmental Science and Pollution Research International. 20, 25462555.

Muyzer, G., Dewaal, E.C., Uitterlinden, A.G. 1993. Profiling of Complex MicrobialPopulations by Denaturing Gradient GelElectrophoresis Analysis of Polymerase Chain Reaction-Amplified Genes-Coding for 16s Ribosomal-Rna. Applied Environmental Microbiology. 59, 695-700.

Omirou, M., Dalias, P., Costa, C., Papastefanou, C., Dados, A., Ehaliotis, C., Karpouzas, D.G. 2012. Exploring the potential of biobeds for the depuration of pesticide-contaminated wastewaters from the citrus production chain: laboratory, column and field studies. Environmental Pollution. 166, 31-39.

Reed, J., Kremer, R., Keaster, A. 1987. Characterization of microorganism in soils exhibiting accelerated pesticide degradation. Bulletin of Environmental Contamination and Toxicology. 39, 776-782.

Sniegowski, K., Bers, K., Van Goetem, K., Ryckeboer, J., Jaeken, P., Spanoghe P., Springael, D. 2011. Improvement of pesticide mineralization in on-farm biopurification systems by bioaugmentation with pesticideprimed soil, Fems Microbiology Ecology. 76, 64-73. 
Spliid, N.H., Helweg, A., Heinrichson, K. 2006. Leaching and degradation of 21 pesticides in a full-scale model biobed. Chemosphere. 65, 2223-2232.

Torstensson, L., Castillo, M.d.P. 1997. Use of biobeds in Sweden to minimize environmental spillages from agricultural spraying equipment. Pesticide Outlook. 8, 24-27.

Tortella, G.R, Rubilar, O, Cea, M, Wulff, C, Martínez, O, \& Diez, M.C. (2010). Biostimulation of Agricultural Biobeds with NPK Fertilizer on Chlorpyrifos Degradation to Avoid Soil and Water Contamination. Journal of soil science and plant nutrition, 10(4), 464-475.

Tortella, G.R., Rubilar, O., Castillo, M.d.P., Cea, M., Mella-Herrera, R., Diez, M.C. 2012. Chlorpyrifos degradation in a biomixture of biobed at different maturity stages. Chemosphere. 88, 224-228.

Tortella, G.R, Mella-Herrera, R.A, Sousa, D.Z, Rubilar, O, Acuña, J.J, Briceño, G, Diez MC. 2013a. Atrazine dissipation and its impact on the microbial communities and community level physiological profiles in a microcosm simulating the biomixture of on-farm biopurification system. Journal Hazardouz Material. 260, 459-467.

Tortella, G.R, Mella-Herrera, R.A, Sousa, D.Z, Rubilar, O, Briceño, G., Parra, L., Diez M.C. 2013b. Carbendazim dissipation in the biomixture of on-farm biopurification system and its effect on microbial communities. Chemosphere. 93, 1084-1093.
Triky-Dotan, S., Ofek, M., Austerweil, M., Steiner, B., Minz, D., Katan, J., and Gamliel, A. 2010. Microbial aspects of accelerated degradation of metam sodium in soil. Phytopathology. 100, 367-375.

Vainio, E.J., Hantula, J. 1999. Variation of RAMS markers within the intersterility group of Heterobasidion annosum in Europe. European Journal of Forest Pathology. 29, 231-246.

Vischetti, C., Coppola, L., Monaci, E., Cardinali, A. \& Castillo, M.d.P. 2007. Microbial impact of the pesticide chlorpyrifos in Swedish and Italian biobeds. Agronomy for Sustainable Development. 27, 267-272.

Vischetti, C., Monaci, E., Cardinali, A., Casucci, C., Perucci, P. 2008. The effect of initial concentration, co-application and repeated applications on pesticide degradation in a biobed mixture. Chemosphere. 72, 17391743 .

White, T., Bruns, T., Lee, S., Taylor, J. 1990. Amplification and direct sequencing of fungal ribosomal RNA genes for phylogenetics. In: Innis, M., Gelfand, D., Shinsky, J., White, T. (Eds.). PCR Protocols: A Guide to Methods and Applications. Academic Press. pp. 315322.

Yu, Y., Chu, X., Pang, G., Xiang, Y., Fang, H. 2009. Effects of repeated applications of fungicide carbendazim on its persistence and microbial community in soil. Journal of Environmental Science. 21, 179-185. 